\title{
GSC-based equalizer with QR decomposition for CP-Free MIMO-OFDM systems
}

\author{
Masakazu Kizawa ${ }^{1}$ and Tetsushi Ikegami ${ }^{1}$ \\ ${ }^{1}$ Graduate School of Science and Technology, Meiji University \\ 1-1-1, Higashimita, Tama-ku, Kawasaki, Kanagawa 214-8571, Japan \\ 1) $\{$ kizawa,ikegami\}@meiji.ac.jp
}

\begin{abstract}
In $\mathrm{CP}($ Cyclic Prefix)-Free OFDM systems, an occurrence of IBI(Inter-Block-Interference) is inevitable due to the loss of CP. Since it deteriorates the system performance, a GSC(Generalized Sidelobe Canceller)-based equalizer for SIMO(MIMO)-OFDM systems have been proposed. In the equalizer, a set of basis of null space of channel matrix has to be found. In this letter, we propose a GSC equalizer using QR decomposition for CP-Free MIMO-OFDM systems, where the reduced complexity maximum likelihood detection by $\mathrm{QR}$ M-algorithm(QRM-MLD) can be applied after the equalization. By computer simulations, we show the BER performance can be improved as compared to the conventional GSC equalizer with the V-BLAST detection.
\end{abstract}

Keywords: CP-Free OFDM, MIMO-OFDM, Generalized Sidelobe Canceller, QRM-MLD, QR decomposition

Classification: Wireless communication technologies

\section{References}

[1] S. Trautmann and N. J. Fliege, "A new equalizer for multitone systems without guard time," IEEE Commun. Lett., vol. 6, pp. 34-36, jan 2002.

[2] C. Y. Lin, J. Y. Wu, and T. S. Lee, "Gsc-based frequency-domain equalizer for cp-free ofdm systems," IEEE International Conf. on Commun., pp. 1132-1136, may 2005.

[3] C. Y. Lin, J. Y. Wu, and T. S. Lee, "An efficient interference cancellation scheme for cp-free mimo-ofdm systems," IEEE VTC-2005-Fall, pp. 11321136, sep 2005.

[4] C. Y. Lin, J. Y. Wu, and T. S. Lee, "A near-optimal low-complexity transceiver for cp-free multi-antenna ofdm systems," IEEE Trans. Commun., vol. E89-B, no. 1, jan 2006.

[5] Y. S. Cho, MIMO-OFDM Wireless Communications with MATLAB, Wiley-IEEE Press, 2010.

\section{Introduction}

OFDM transmission, $\mathrm{CP}$ is prepended to each OFDM symbol in order to leviate the effect of multipath channel. However, since the CP is redun- 
dant and it degrades spectrum efficiency, CP-Free OFDM systems has been considered[1, 2, 3, 4]. Due to the loss of CP, an occurrence of IBI(InterBlock-Interference), which consists of an ISI(Inter-Symbol-Interference) and ICI(Inter-Carrier-Interference), is inevitable. Since it deteriorates the system performance, an IBI compensation is essential in those system. Lin et al. $[2,3,4]$ has been proposed a GSC-based equalizer for CP-Free SIMO(MIMO)OFDM systems. In this letter, we propose the GSC-based equalizer using QR decomposition to construct the blocking matrix, where the reduced complexity MLD, such as QRM-MLD [5], can be applied as the data detection on each subcarrier after the equalization. By computer simulations, we show that the additional performance gain in terms of Bit-Error-Rate(BER) is achieved by the proposed GSC equalizer with QRM-MLD as compared to the conventional GSC equalizer with the V-BLAST detection.

Note that $\mathbb{C}$ denotes the complex field. $\mathbf{I}_{N}$ is an unit matrix with the size $N .(\cdot)^{T}$ and $(\cdot)^{H}$ represent the transpose and Hermitian transpose, respectively. Also, $\odot$ represents the Kronecker product.

\section{System Model}

\subsection{CP-Free MIMO-OFDM Systems}

We consider CP-Free MIMO-OFDM systems with $N_{T}$ transmitters, $N_{R}$ receivers and $K$ subcarriers. Let $x_{i, k}(n) \in \mathbb{C}$ be the transmitted symbol at the $i$-th transmit antenna, the $k$-th subcarrier and the $n$-th OFDM symbol. The transmitted symbols $\mathbf{x}_{i}(n) \in \mathbb{C}^{N_{T}}$ at the $i$-th transmit antenna can be written as $\mathbf{x}_{i}(n)=\left[x_{i, 1}(n), \ldots, x_{i, K}(n)\right]^{T}$. Then, the transmitted OFDM symbol $\mathbf{s}_{i}(n) \in \mathbb{C}^{N_{T} K}$ can be represented as

$$
\mathbf{s}_{i}(n)=\mathbf{F}^{H} \mathbf{x}_{i}(n)
$$

where $\mathbf{F} \in \mathbb{C}^{K \times K}$ is the DFT(Discrete-Fourier-Transform) matrix. Letting $\mathbf{x}(n)=\left[\mathbf{x}_{1}^{T}(n), \mathbf{x}_{2}^{T}(n), \ldots, \mathbf{x}_{N_{T}}^{T}(n)\right]^{T} \in \mathbb{C}^{N_{T} K}$ be the stacked transmitted symbols, the stacked transmitted OFDM symbol can be written as

$$
\mathbf{s}(n)=\mathbf{F}_{N_{T}}^{H} \mathbf{x}(n),
$$

where $\mathbf{F}_{N_{T}}^{H}=\mathbf{I}_{N_{T}} \odot \mathbf{F}^{H} \in \mathbb{C}^{N_{T} K \times N_{T} K}$. Assuming that a channel impulse response(CIR) can be modeled as a tapped delay line with $L$ tap, the coefficients of the CIR between the $i$-th transmitter and $j$-th receiver can be expressed as $\mathbf{h}_{j, i}=\left[h_{j, i}(0), h_{j, i}(1), \ldots, h_{j, i}(L-1)\right]^{T} \in \mathbb{C}^{L}$. In the CP-Free OFDM systems, the received OFDM symbol at the $j$-th receive antenna $\mathbf{r}_{j}(n) \in \mathbb{C}^{K}$ can be expressed as

$$
\mathbf{r}_{j}(n)=\sum_{i=1}^{N_{T}}\left\{\mathbf{H}_{0, j, i} \mathbf{s}_{i}(n)+\mathbf{H}_{1, j, i} \mathbf{s}_{i}(n-1)\right\}+\mathbf{w}_{j}(n)
$$

where $\mathbf{w}_{j}(n) \in \mathbb{C}^{K}$ is a noise vector and $\mathbf{H}_{0, j, i} \in \mathbb{C}^{K \times K}$ and $\mathbf{H}_{1, j, i} \in \mathbb{C}^{K \times K}$ are the channel Toeplitz matrix and the channel upper triangular Toeplitz 
matrix, respectively. On the other hand, the received symbols $\mathbf{y}_{j}(n) \in \mathbb{C}^{K}$ can be obtained by premultiplying $\mathbf{F}$ by $\mathbf{r}_{j}(n)$ as

$$
\begin{aligned}
\mathbf{y}_{j}(n) & =\mathbf{F r}_{j}(n) \\
& =\sum_{i=1}^{N_{T}}\left\{\mathbf{D}_{j, i} \mathbf{x}_{i}(n)-\mathbf{C}_{j, i} \mathbf{x}_{i}(n)+\mathbf{S}_{j, i} \mathbf{x}_{i}(n-1)\right\}+\mathbf{z}_{j}(n) \\
& =\sum_{i=1}^{N_{T}}\left\{\mathbf{D}_{j, i} \mathbf{x}_{i}(n)+\mathbf{S}_{j, i} \mathbf{e}_{i}(n)\right\}+\mathbf{z}_{j}(n)
\end{aligned}
$$

where $\mathbf{z}_{j}(n)=\mathbf{F w}_{j}(n), \mathbf{e}_{i}(n)=\mathbf{x}_{i}(n-1)-\mathbf{x}_{i}(n) \in \mathbb{C}^{N_{R} K}, \mathbf{z}_{i}(n)=\mathbf{F w}_{j}(n) \in$ $\mathbb{C}^{N_{R} K}, \mathbf{D}_{j, i}=\mathbf{F}\left(\mathbf{H}_{0, j, i}+\mathbf{H}_{1, j, i}\right) \mathbf{F}^{H} \in \mathbb{C}^{N_{R} K \times N_{T} K}, \mathbf{C}_{j, i}=\mathbf{F H}_{0, j, i} \mathbf{F}^{H}, \mathbf{S}_{j, i}=$ $\mathbf{F H}_{1, j, i} \mathbf{F}^{H}, . \mathbf{D}_{j, i}, \mathbf{S}_{j, i}, \mathbf{C}_{j, i}$ express the channel matrix, ISI matrix and ICI matrix, respectively. Note that $\mathbf{S}_{j, i}=\mathbf{C}_{j, i}$ holds in the case of the CP-Free OFDM systems. Thus, the stacked received symbols $\mathbf{y}(n)=\left[\mathbf{y}_{1}^{T}(n), \ldots, \mathbf{y}_{N_{R}}^{T}(n)\right]^{T} \in \mathbb{C}^{N_{R} K}$ can also be written as

$$
\mathbf{y}(n)=\mathbf{D} \mathbf{x}(n)+\mathbf{S e}(n)+\mathbf{z}(n),
$$

where $\mathbf{e}(n)=\left[\mathbf{e}_{1}^{T}(n), \ldots, \mathbf{e}_{N_{T}}^{T}(n)\right]^{T} \in \mathbb{C}^{N_{T} K}, \mathbf{w}(n)=\left[\mathbf{w}_{1}^{T}(n), \ldots, \mathbf{w}_{N_{R}}^{T}(n)\right]^{T} \in$ $\mathbb{C}^{N_{R} K}$. Also, $\mathbf{D}$ and $\mathbf{S}$ are the total channel matrix and ISI matrix having $\mathbf{D}_{j, i}, \mathbf{S}_{j, i}$ as the $(j, i)$ element block, respectively.

\subsection{GSC-based Equalizer with QR decomposition}

The GSC-based equalizer consists of three matrices, a coherent matrix $\mathbf{Q}$, blocking matrix $\mathbf{B}$ and weight matrix $\mathbf{U}$. In addition, a dimension reduction matrix $\mathbf{T}$ is also introduced in the partial adaptive GSC equalizer. Fig. $\mathbf{1}$ shows the block diagram of the partial adaptive GSC equalizer.

First of all, we describe the construction of the blocking matrix. The blocking matrix $\mathbf{B}$ has to be orthogonal to the channel matrix $\mathbf{D}$, namely, $\mathbf{B}^{H} \mathbf{D}=\mathbf{O}$ and each column of $\mathbf{B}$ must be orthonormal to each other. Thus, it turns out that each column of $\mathbf{B}$ is the orthonormal basis in the left null space of the $\mathbf{D}$. Let $\mathbf{D}_{k} \in \mathbb{C}^{N_{R} \times N_{T}}$ be the partial channel matrix on the $k$-th subcarrier which is made of the $k$-th diagonal element of each $\mathbf{D}_{j, i}$. The $(j, i)$ element of $\mathbf{D}_{k}$ is denoted by $D_{m, i, j}=D_{k+K(j-1), k+K(i-1)}$. Given $\mathbf{b}_{k} \in \mathbb{C}^{N_{R}}$ as one of the basis in the left null space of $\mathbf{D}_{k}, \mathbf{b}_{k}$ can be extended into

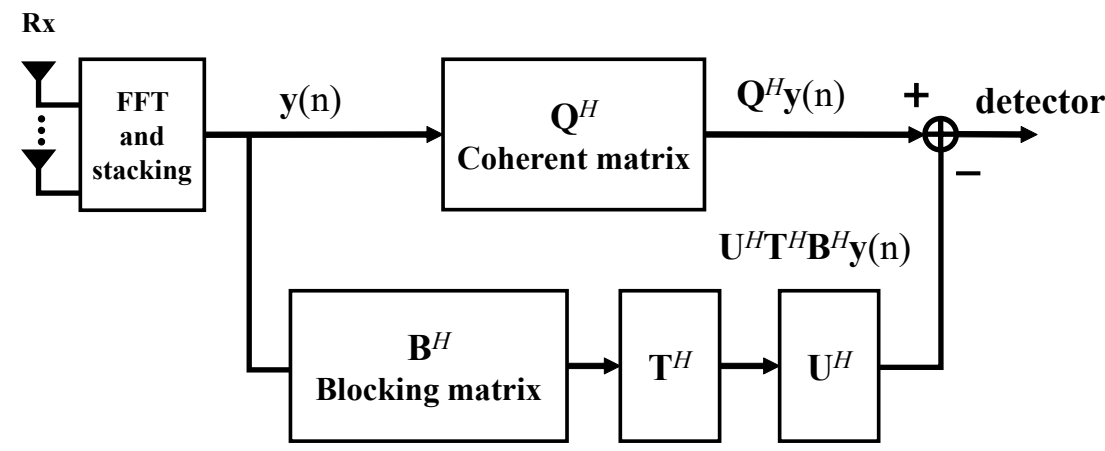

Fig. 1: The block diagram of the proposed partial adaptive GSC equalizer. 
$\mathbf{b}_{k}^{\prime} \in \mathbb{C}^{N_{R} K}$ by zero insertion, which is in the left null space of $\mathbf{D}$. The $m$-th element $b_{k, m}^{\prime}$ of $\mathbf{b}_{k}^{\prime}$ is given as

$$
b_{k, m}^{\prime}=\left\{\begin{array}{ll}
b_{k, j}, & \text { for } m=k+K(j-1), \quad 1 \leq j \leq N_{R} \\
0, & \text { otherwise }
\end{array},\right.
$$

where $b_{k, j}$ is the $j$-th element of $\mathbf{b}_{k}$. Hence, a set of basis of the left null space has to be found on each $\mathbf{D}_{k}$. While gaussian elimination can be utilized to find the bases, those can also be obtained by QR decomposition. When $N_{R}>N_{T}$ and each $\mathbf{D}_{k}$ has column full rank, the size of dimension of the left null space of $\mathbf{D}_{k}$ is provided by $N_{R}-N_{T}$. Let $\mathbf{D}_{k}=\mathbf{Q}_{k} \mathbf{R}_{k}$ be the QR decomposition of $\mathbf{D}_{k}$, where $\mathbf{Q}_{k} \in \mathbb{C}^{N_{R} \times N_{R}}$ is an unitary matrix and $\mathbf{R}_{m} \in \mathbb{C}^{N_{R} \times N_{T}}$ is an upper triangular matrix. Then, splitting $\mathbf{Q}_{k}$ as $\mathbf{Q}_{k}=\left[\mathbf{Q}_{k, 0}, \mathbf{Q}_{k, 1}\right]$, where $\mathbf{Q}_{k, 0}=$ $\left[\mathbf{q}_{k, 1}, \ldots \mathbf{q}_{k, N_{T}}\right] \in \mathbb{C}^{N_{R} \times N_{T}}$ and $\mathbf{Q}_{k, 1}=\left[\mathbf{q}_{k, N_{T}+1}, \ldots, \mathbf{q}_{k, N_{R}}\right] \in \mathbb{C}^{N_{R} \times\left(N_{R}-N_{T}\right)}$, a set of orthonormal basis of the null space of $\mathbf{D}_{m}$ as $\mathbf{Q}_{k, 1}$. Let $\mathbf{Q}_{k}^{\prime}=\mathbf{Q}_{k, 0}$, $\mathbf{B}_{k}=\mathbf{Q}_{k, 1}$ and let $\mathbf{Q}_{k}^{\prime \prime} \in \mathbb{C}^{N_{R} K \times N_{T}}, \mathbf{B}_{k}^{\prime} \in \mathbb{C}^{\left(N_{R}-N_{T}\right) M \times N_{T}}$ be the matrices which are obtained by extending each column vector of $\mathbf{Q}_{k}^{\prime}, \mathbf{B}_{k}$ as in Eq. (6), respectively. Consequently, letting $\mathbf{Q}=\left[\mathbf{Q}_{1}^{\prime \prime}, \ldots, \mathbf{Q}_{K}^{\prime \prime}\right] \in \mathbb{C}^{N_{R} K \times N_{T} K}$ and $\mathbf{B}=$ $\left[\mathbf{B}_{1}^{\prime}, \ldots, \mathbf{B}_{K}^{\prime}\right] \in \mathbb{C}^{N_{R} K \times\left(N_{R}-N_{T}\right) M}$, the coherent matrix and blocking matrix are obtained.

Subsequently, the weight matrix can be obtained by solving the following mean square error:

$$
J(\mathbf{U})=\mathrm{E}\left[\left\|\mathbf{Q}^{H} \mathbf{y}(n)-\mathbf{U}^{H} \mathbf{T}^{H} \mathbf{B}^{H} \mathbf{y}(n)\right\|^{2}\right],
$$

where $\mathbf{T} \in \mathbb{C}^{\left(N_{R}-N_{T}\right) K \times N_{T} L}$ can be composed of $\mathbf{B}, \mathbf{F}$ and $h_{j, i}(l)$. For more details, please refer to [3]. Solving the minimization of Eq. (7), the optimum weight matrix $\mathbf{U}_{\mathrm{opt}}$ is given as

$$
\mathbf{U}_{\text {opt }}=\left(\mathbf{T}^{H} \mathbf{B}^{H} \boldsymbol{\Sigma}_{\text {in }} \mathbf{B} \mathbf{T}\right)^{-1} \mathbf{T}^{H} \mathbf{B}^{H} \boldsymbol{\Sigma}_{\text {in }} \mathbf{Q},
$$

where $\boldsymbol{\Sigma}_{\text {in }} \in \mathbb{C}^{N_{T} K \times N_{T} K}$ is a covariance matrix given by

$$
\begin{aligned}
\boldsymbol{\Sigma}_{\text {in }} & =\mathrm{E}\left[\mathbf{v}(n) \mathbf{v}^{H}(n)\right] \\
& =\mathbf{S E}\left[\mathbf{e}(n) \mathbf{e}^{H}(n)\right] \mathbf{S}^{H}+\mathrm{E}\left[\mathbf{z}(n) \mathbf{z}^{H}(n)\right] \\
& =2 \sigma_{\mathrm{s}}^{2} \mathbf{S S}^{H}+\sigma_{n}^{2} \mathbf{I},
\end{aligned}
$$

where $\mathbf{v}(n)=\mathbf{S e}(n)+\mathbf{z}(n), \mathrm{E}\left[\mathbf{e}(n) \mathbf{e}^{H}(n)\right]=2 \sigma_{\mathrm{s}}^{2} \mathbf{I}_{N_{T} K}, \mathrm{E}\left[\mathbf{z}(n) \mathbf{z}^{H}(n)\right]=$ $\sigma_{\mathrm{n}}^{2} \mathbf{I}_{N_{R} K} \cdot \sigma_{\mathrm{s}}^{2}$ and $\sigma_{\mathrm{n}}^{2}$ express average signal power and noise variance, respectively. We assume that the transmitted symbols are uncorrelated each other. Then, the stacked received symbols after the equalization $\tilde{\mathbf{y}}(n) \in \mathbb{C}^{N_{T} K}$ can be expressed as

$$
\begin{aligned}
\tilde{\mathbf{y}}(n) & =\mathbf{Q}^{H} \mathbf{y}(n)-\mathbf{Q}^{H} \mathbf{U}^{H} \mathbf{T}^{H} \mathbf{B}^{H} \mathbf{y}(n) \\
& =\mathbf{Q}^{H}\left(\mathbf{I}_{N_{R} K}-\mathbf{U}^{H} \mathbf{T}^{H} \mathbf{B}^{H}\right) \mathbf{y}(n) \\
& =\mathbf{W}^{H} \mathbf{y}(n) \\
& =\tilde{\mathbf{R}} \mathbf{x}(n)+\tilde{\mathbf{z}}(n),
\end{aligned}
$$


where $\mathbf{W}=\left(\mathbf{I}_{N_{R} K}-\mathbf{B T U}\right) \mathbf{Q} \in \mathbb{C}^{N_{R} K \times N_{T} K}, \tilde{\mathbf{R}}=\mathbf{Q}^{H} \mathbf{D} \in \mathbb{C}^{N_{T} K \times N_{T} K}$ and $\tilde{\mathbf{z}}(n)=\mathbf{W}^{H}\{\mathbf{S e}(n)+\mathbf{z}(n)\} \in \mathbb{C}^{N_{T} K}$ is the residual noise vector. The received symbols on the $k$-th subcarrier $\tilde{\mathbf{y}}_{k}(n)=\left[\tilde{y}_{k}(n), \ldots, \tilde{y}_{k+\left(N_{R}-1\right) K}(n)\right]^{T} \in$ $\mathbb{C}^{N_{T}}$ can be written as

$$
\tilde{\mathbf{y}}_{k}(n)=\tilde{\mathbf{R}}_{k} \mathbf{x}_{k}(n)+\tilde{\mathbf{z}}_{k}(n)
$$

where $\tilde{\mathbf{R}}_{k} \in \mathbb{C}^{N_{T} \times N_{T}}$ is the partial matrix corresponding to the top $N_{T}$ rows of $\mathbf{R}_{m} . \quad \mathbf{x}_{k}(n)=\left[x_{1, k}(n), \ldots, x_{N_{T}, k}(n)\right]^{T} \in \mathbb{C}^{N_{T}}$ and $\tilde{\mathbf{z}}_{k}(n) \in \mathbb{C}^{N_{T}}$ are the transmitted symbols and residual noise on the $k$-th subcarrier, respectively. Consequently, the QRM-MLD can be applied to $\tilde{\mathbf{y}}_{k}(n)$.

\section{Computer Simulations}

First, we describe the simulation settings. The CP-Free MIMO-OFDM system with 64 subcarriers is considered. For data modulation, QPSK is used. For channel model, an exponential decay channel with 9-tap, $p[l]=$ $1 / P \exp \left(-l / \sigma_{\tau}\right)$, where $\sigma_{\tau}$ and $P$ denote an normalized delay spread and normalization constant, is used. We assume that each path follows the i.i.d Rayleigh fading and the timing synchronization and channel estimation are ideal. Fig. 2 shows the results of BER performance in terms of $E_{b} / N_{0}$, where $E_{b}$ is an energy per bit and $N_{0}$ is noise power density. The difference between Fig. 2a and Fig. 2b is the number of receive antennas $N_{R}$ while the number of transmit antennas $N_{T}$ are the same. In the figure, "QRM-MLD" indicates the result of the proposed partial adaptive GSC equalizer with the QRMMLD, where "M" denotes the number of survived paths in the M-algorithm. For comparison, the results of the conventional GSC equalizer with the VBLAST detection based on a MMSE(minimum mean square error) criterion and the MLD are shown, respectively. In addition, the result of CP-OFDM systems with MLD, which is referred as "MLD(SCP)", is also shown. When $M=1$, the result of QRM-MLD shows a poor performance as compared to the conventional GSC equalizer with the V-BLAST detection, however, when $M=2$, the result shows the identical or better performance. When $M=4$, the result matches the conventional GSC equalizer with MLD. As a result, the additional performance gain about $1.5 \sim 2.0 \mathrm{~dB}$ can be achieved as compared to the conventional one.

\section{Conclusions}

In this letter, we proposed the GSC-based equalizer using QR decomposition to construct the blocking matrix for MIMO-OFDM systems, where the reduced complexity MLD, such as QRM-MLD, can be applied as the data detection for the equalized symbols on each subcarrier. By computer simulations, the additional improvement can be achieved in terms of BER performance by the proposed GSC-based equalizer and the QRM-MLD. 


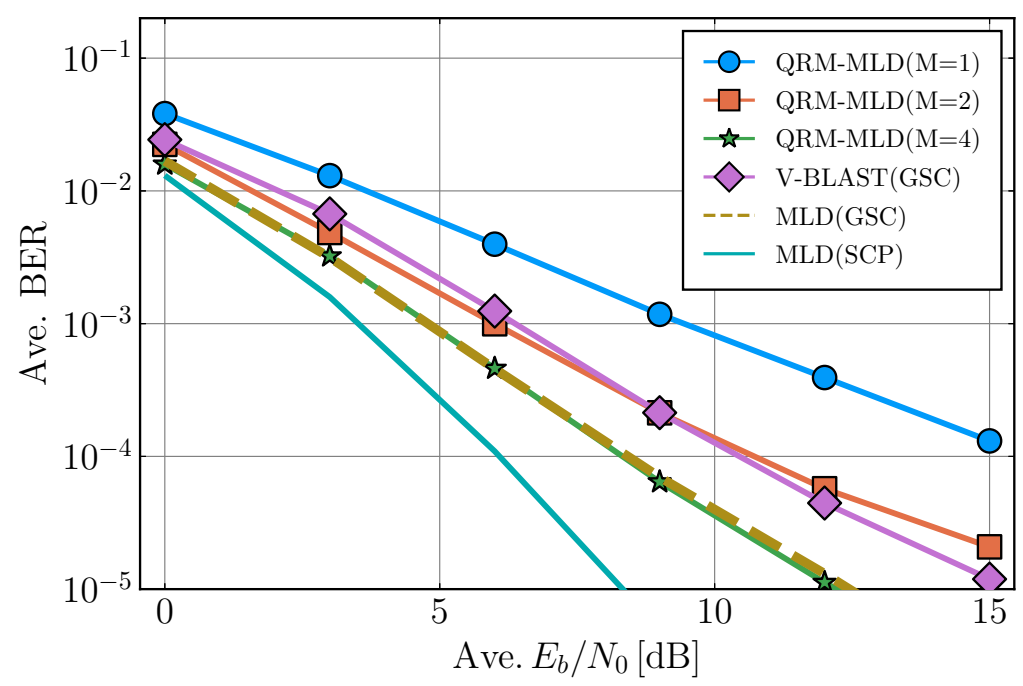

(a) $N_{T}=4, N_{R}=5$

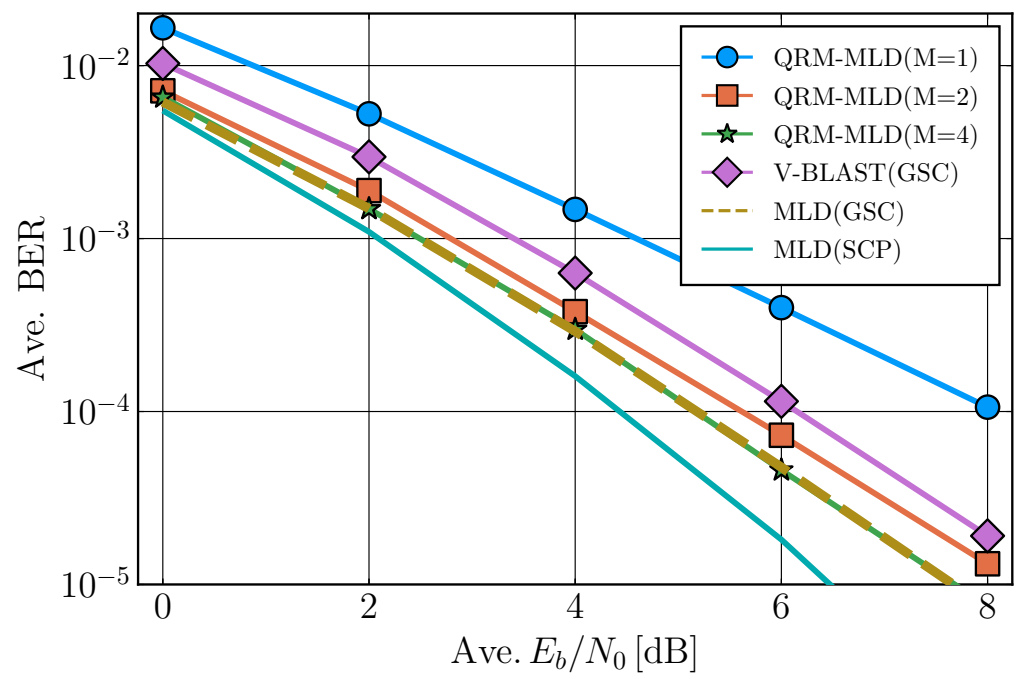

(b) $N_{T}=4, N_{R}=6$

Fig. 2: The results of average $E_{b} / N_{0}$ v.s. average BER in each transceivers. 